Published in final edited form as:

N Engl J Med. 2004 January 29; 350(5): 443-450.

\title{
Human Metapneumovirus and Lower Respiratory Tract Disease in Otherwise Healthy Infants and Children
}

\author{
John V. Williams, M.D., Paul A. Harris, Ph.D., Sharon J. Tollefson, B.A., Lisa L. Halburnt-Rush, \\ M.Ed., Joyce M. Pingsterhaus, B.A., Kathryn M. Edwards, M.D., Peter F. Wright, M.D., and \\ James E. Crowe Jr., M.D. \\ From the Division of Infectious Diseases, Departments of Pediatrics, (J.V.W., S.J.T., L.L.H.-R., \\ J.M.P., K.M.e., P.F.W., J.E.C.), Biomedical Engineering (P.A.H.), and Microbiology and Immunology \\ (P.F.W., J.E.C.), Vanderbilt University Medical Center, Nashville
}

\begin{abstract}
BACKGROUND-We sought to determine the role of human metapneumovirus in lower respiratory tract illness in previously healthy infants and children.

METHODS-We tested nasal-wash specimens, obtained over a 25-year period from otherwise healthy children presenting with acute respiratory tract illness, for human metapneumovirus.

RESULTS-A viral cause other than human metapneumovirus was determined for 279 of 687 visits for acute lower respiratory tract illness (41 percent) by 463 children in a population of 2009 infants and children prospectively seen from 1976 to 2001 . There were 408 visits for lower respiratory tract illness by 321 children for which no cause was identified. Of these 321 children, specimens from 248 were available. Forty-nine of these 248 specimens ( 20 percent) contained human metapneumovirus RNA or viable virus. Thus, 20 percent of all previously virus-negative lower respiratory tract illnesses were attributable to human metapneumovirus, which means that 12 percent of all lower respiratory tract illnesses in this cohort were most likely due to this virus. The mean age of human metapneumovirus-infected children was 11.6 months, the male:female ratio was 1.8:1, 78 percent of illnesses occurred between December and April, and the hospitalization rate was 2 percent. The virus was associated with bronchiolitis in 59 percent of cases, pneumonia in 8 percent, croup in 18 percent, and an exacerbation of asthma in 14 percent. We also detected human metapneumovirus in 15 percent of samples from 261 patients with upper respiratory tract infection but in only 1 of 86 samples from asymptomatic children.
\end{abstract}

CONCLUSIONS-Human metapneumovirus infection is a leading cause of respiratory tract infection in the first years of life, with a spectrum of disease similar to that of respiratory syncytial virus.

Respiratory Syncytial Virus (RSV), parainfluenza virus, adenovirus, and influenzavirus are common known causes of lower respiratory tract disease in infants and children. ${ }^{1-3}$

Nevertheless, in a substantial portion of lower respiratory tract infections in children, no virus can be cultured. In 2001, researchers in the Netherlands isolated a new virus from children and adults with acute respiratory tract infection. ${ }^{4}$ This RNA virus, provisionally designated human metapneumovirus, is closely related to avian pneumovirus. Since then, investigators in Canada, Australia, the United Kingdom, and the United States have described patients with acute

Address reprint requests to Dr. Crowe at Vanderbilt University Medical Center, D-7235 Medical Center N., 1161 21st Ave. S., Nashville, TN 37232-2581, or at james.crowe@ vanderbilt.edu..

We are indebted to Yuwei Zhu, M.D., and Bonnie LaFleur, Ph.D., for statistical support; to Sandra Yoder and Mine Ikizler for their assistance with laboratory studies; to Deb Macheca for secretarial support; and to William Schaffner, M.D., and Marie Griffin, M.D., for helpful comments. 
respiratory tract infection due to human metapneumovirus. ${ }^{5-8}$ We sought to define the etiologic role of this virus in lower respiratory tract infections in previously healthy young children.

\section{METHODS STUDY DESIGN}

The study was conducted at the Vanderbilt Vaccine Clinic in Nashville, a primary care clinic established to evaluate investigational vaccines in young children and conduct surveillance for respiratory viruses. ${ }^{9-12}$ Healthy, full-term infants were enrolled at birth and followed for up to five years. Children in whom chronic diseases developed, other than mild asthma, were excluded. All visits were conducted within the General Clinical Research Center, with care provided by the pediatric infectious diseases faculty and nurse practitioners. During visits for illness, the children's signs and symptoms were recorded on a standardized clinical form, the information was reviewed and entered into a data base, and nasal-wash samples were obtained and cultured for viruses. All studies were conducted with the approval of the Committee for the Protection of Human Subjects of the Vanderbilt University Medical Center. Parents gave written informed consent.

\section{CASE DEFINITIONS}

The clinician made the diagnosis at the time of the child's visit. Wheezing, rales, tachypnea, and dyspnea were considered to be signs of lower respiratory tract infection. Bronchiolitis was defined as an acute respiratory illness characterized by rhinorrhea, cough, and diffuse wheezes and rales, with peribronchial thickening and hyperexpansion on the chest radiograph, if one was obtained. Pneumonia was defined as dyspnea in a patient with focal rales or decreased breath sounds and the presence of a focal infiltrate on the chest radiograph. Laryngotracheobronchitis (croup) was defined as an acute lower respiratory tract infection characterized by hoarseness, cough, and stridor. Nasal-wash specimens for viral culture were obtained from children with any of the following indications: an upper respiratory tract infection accompanied by a temperature greater than $38.4^{\circ} \mathrm{C}$, acute otitis media, or evidence of a lower respiratory tract infection. Specimens were kept on ice and promptly inoculated onto cell-culture monolayers, including human neonatal kidney, human embryonic lung, HEp-2, rhesus-monkey kidney, and (during influenza season) Madin-Darby canine kidney. Aliquots of nasal washes were snap-frozen and stored at $-70^{\circ} \mathrm{C}$.

\section{STUDY GROUPS}

Children who switched from the clinic to community-based care were replaced by newborns to maintain a population of approximately 200 children. During the study period, from 1976 to 2001, 2009 children were followed in the clinic. We studied specimens from patients given a diagnosis of upper or lower respiratory tract infection who had previously negative viral cultures. We also tested specimens from patients who had a lower respiratory tract infection with cultures that were positive for viruses other than human metapneumovirus. Samples from children without respiratory symptoms, collected immediately before enrollment in vaccine trials, were tested for human metapneumovirus to assess the rate of asymptomatic infection.

\section{MOLECULAR ANALYSIS}

Nasal-wash specimens were thawed at $37^{\circ} \mathrm{C}$, and RNA was extracted with the use of the QIAamp Viral RNA kit (Qiagen). Reverse transcription (RT) was performed with the use of random hexamer primers. Polymerase-chain-reaction (PCR) assays were performed in duplicate. Primers amplified a 170-bp fragment of the $L$ (polymerase) gene, which is highly conserved among isolates of human metapneu-movirus. ${ }^{4}$ Products were gel-purified and 
cloned into a commercial plasmid vector (Promega). The sequences of both strands of complementary DNA from the insert were determined on an ABI 377 Prism instrument in the Vanderbilt DNA Sequencing Core Laboratory. Samples were considered to be positive if they had a unique sequence or if the results of both PCR assays were positive. Extracted RNA was also tested by multiplex RT-PCR for RSV, parainfluenza virus, and influenzavirus with the use of an established method (Hexaplex, Prodesse). ${ }^{13}$ Sequences were aligned with each other and with published sequences of human metapneumovirus (GenBank accession numbers AF371330 through AF371338) with the use of ClustalW alignment in MacVector (Accelrys). Phylogenetic analysis was performed with the use of Phylip software, version 3.5. ${ }^{14}$

\section{CULTURE OF HUMAN METAPNEUMOVIRUS ISOLATES}

Samples were inoculated onto monolayers of Vero and LLC-MK2 cells in OptiMEM medium (Invitrogen) with trypsin but without serum and were assessed three times a week. Wells with a cytopathic effect, consisting of rounded cells and focal plaques, were tested for the presence of human metapneumovirus by RT-PCR. Cultures without a cytopathic effect were passaged onto fresh cell monolayers after 14 days and incubated for an additional 14 days.

\section{STATISTICAL ANALYSIS}

We used the chi-square test to compare samples that were tested with those not tested with respect to the monthly distribution of visits, sex, and the percentage of samples associated with a specific diagnosis (i.e., bronchiolitis, croup, pneumonia, or an exacerbation of asthma). We used two-tailed t-tests to compare the mean ages of the two groups. The random subgroup of patients with upper respiratory tract infections was selected with the use of a randomization routine in SAS software, version 8.2 (SAS Institute), based on random numbers generated from a uniform distribution. This subgroup of specimens (one from each patient) was compared with the entire group of specimens from patients with upper respiratory tract infections that were negative for virus with the use of the methods described above. Symptoms associated with different viruses were compared with the use of Fisher's exact test, and the mean age at the onset of illness was compared in these groups with the use of the Mann-Whitney test owing to the skewed distribution of data. Analyses were performed with the use of SPSS for Windows, version 10.0.5. All reported $\mathrm{P}$ values are two-sided.

\section{RESULTS \\ DEMOGRAPHIC CHARACTERISTICS}

During the 25-year study period, 2009 children were enrolled, with 5061 child-years of followup (average follow-up, 2.5 years). Seventeen percent of the child-years were accounted for by infants younger than 6 months of age, 15 percent by children 6 to 12 months of age, 25 percent by children 13 to 24 months of age, 19 percent by children 25 months to 3 years of age, and 24 percent by children older than 3 years of age. Fifty-one percent of the infants enrolled were male, 53 percent were white, and 44 percent were black.

A total of 1127 visits were associated with the diagnosis of lower respiratory tract infection; at 687 of these visits ( 61 percent) nasal-wash samples were obtained for culture. The 440 visits for lower respiratory tract infection at which cultures were not obtained did not differ significantly from those in which cultures were obtained, with respect to the mean age at the onset of illness, sex, seasonal distribution, or the spectrum of clinical diagnoses. Viral cultures identified 103 patients with RSV, 58 with parainfluenza virus, 32 with influenzavirus, 28 with adenovirus, and 50 with enterovirus, rhinovirus, poliovirus, herpes simplex virus, or rotavirus. Five RSV-infected children were coinfected with other viruses: one each with influenzavirus, adenovirus, and parainfluenza virus and two with enterovirus. Thus, 408 of 687 nasal-wash specimens from 321 children were previously negative for viruses by culture. Of these 321 
children, 248 had samples remaining for RT-PCR analysis. When these samples were compared with the 160 samples that were not available for testing, they did not differ significantly with respect to the mean age at the onset of illness, sex, seasonal distribution, or spectrum of clinical diagnoses.

\section{LOWER RESPIRATORY TRACT INFECTION WITH HUMAN METAPNEUMOVIRUS}

Forty-nine of the 248 tested samples (20 percent) were positive for human metapneumovirus by RT-PCR. Of these, 22 isolates were recovered in culture and confirmed by the presence of a cytopathic effect and by RT-PCR for human metapneumovirus genes from passaged cultures. Of the 49 samples that were positive for human metapneumovirus, 3 were also positive for RSV on RT-PCR, for a coinfection rate of 6 percent. Specimens from 96 patients with lower respiratory tract infection who had previously had positive cultures for other viruses were tested for human metapneumovirus by RT-PCR. Four were also positive for human metapneumovirus, yielding a coinfection rate for these samples of 4 percent. There was no apparent difference in the severity of disease between children with coinfections and those infected with human metapneumovirus alone. Of 86 nasal-wash specimens from children without respiratory symptoms that were tested, only 1 was positive for human metapneumovirus.

The male:female ratio among patients with lower respiratory tract infection due to human metapneumovirus was 1.8:1, with a mean age of 11.6 months, a median age of 6.5 months, and an age range of 1.5 to 50 months. Lower respiratory tract infection with human metapneumovirus occurred predominantly in the first year of life, and the age distribution thus differed from that of the clinic population: 25 percent of human metapneumovirus infections occurred among infants under six months of age, and 49 percent occurred among infants six months to one year of age. The peak number of human metapneumovirus infections was in March, with 38 illnesses (78 percent) occurring between December and April (Fig. 1).

Genetically distinct strains of human metapneumovirus sometimes circulated during the same year (Fig. 2). There were yearly variations in the percentage of lower respiratory tract infections that were negative for other viruses and attributable to human metapneumovirus, ranging from 0 percent to 31 percent in a given year.

Clinical Features-The clinical features on presentation among the children with human metapneumovirus infection of the lower respiratory tract are summarized in Table 1 . The mean duration of symptoms before medical attention was sought was 4.4 days.

The clinical diagnosis given to the 49 children with human metapneumovirus infection of the lower respiratory tract was bronchiolitis in 29 (59 percent), croup in 9 (18 percent), pneumonia in 4 (8 percent), and an exacerbation of asthma in 7 (14 percent) (Table 2). Acute otitis media was diagnosed in 18 (37 percent). Fourteen children underwent chest radiography, and the results were abnormal in seven (50 percent), with the radiographs in most of these children showing diffuse perihilar infiltrates (Fig. 3). One of the 49 children ( 2 percent), who was 36 months of age, was hospitalized with the diagnosis of an exacerbation of asthma triggered by a viral respiratory tract infection.

Comparison with Other Viral Infections-Clinical data for children with lower respiratory tract infections caused by RSV, parainfluenza virus, influenzavirus, or adenovirus were compared with those for children with human metapneumovirus infections. Fever occurred less often with human metapneumovirus infection than with influenzavirus infection (52 percent vs. 87 percent, $\mathrm{P}=0.001$ ) but at a rate similar to that for infections with RSV, parainfluenza virus, or adenovirus. Vomiting occurred less often with human metapneumovirus infection than with RSV infection (in 10 percent of children vs. 31 percent, 
$\mathrm{P}=0.005$ ) or influenzavirus infection (10 percent vs. 28 percent, $\mathrm{P}=0.04)$. On physical examination, rales and wheezing were noted less often in children with human metapneumovirus infection than in those with RSV infection ( 8 percent vs. 24 percent, $\mathrm{P}=0.03$, and 52 percent vs. 69 percent, $\mathrm{P}=0.04$, respectively), but at a rate similar to that for parainfluenza virus, influenzavirus, or adenovirus infection. Conjunctivitis was noted less frequently with human metapneumovirus infection than with influenzavirus infection (4 percent vs. 22 percent, $\mathrm{P}=0.02$ ), whereas the rates of rhinitis and rhonchi were similar among the viruses.

The distribution of specific clinical syndromes caused by human metapneumovirus differed from that of the other viruses (Table 2). Human metapneumovirus infection was more likely to be associated with clinical bronchiolitis and less likely to be associated with croup than was infection with parainfluenza virus or influenzavirus. Human metapneumovirus infection was less likely to be associated with pneumonia than was infection with RSV or influenzavirus. There was an association between human metapneumovirus infection and the diagnosis of an exacerbation of asthma. There were no significant differences in the rates of abnormal chest radiographs, hospitalization, or visits to the emergency room according to the virus.

\section{GENETIC VARIABILITY OF HUMAN METAPNEUMOVIRUS}

Twenty-two of the human metapneumovirus sequences were unique, with distinct genotypes present in different years. The isolates fell into two major clades (Fig. 2). Other studies have also identified two major diversity groups. ${ }^{4,15}$ There were no significant differences in the mean age at the onset of illness, symptoms, or diagnoses associated with viruses in different clades.

\section{UPPER RESPIRATORY TRACT INFECTION WITH HUMAN METAPNEUMOVIRUS}

There were 2326 visits associated with a diagnosis of upper respiratory tract infection and a negative viral culture; a random sample of 261 of the patients was selected as described in the Methods section. The subgroup of 261 patients did not differ significantly from the entire group with respect to the mean age at the onset of illness, sex, the year of the visit, or seasonal distribution. Thirty-nine of these patients (15 percent) were positive for human metapneumovirus. The seasonal distribution of the onset of illness in these patients was similar to that among patients with lower respiratory tract infection caused by human metapneumovirus, whereas the mean age was 19.6 months — older than that of patients with lower respiratory tract infection caused by human metapneumovirus $(\mathrm{P}=0.003)$ but not significantly different from the mean age of all patients with upper respiratory tract infections $(\mathrm{P}=0.39)$. The male:female ratio among patients with upper respiratory tract infection caused by human metapneumovirus was 0.9:1.

\section{RECURRENT INFECTION WITH HUMAN METAPNEUMOVIRUS}

Three patients had evidence of recurrent infection with human metapneumovirus; genetically distinct isolates were obtained at different times. A boy was given a diagnosis of bronchiolitis at 3.5 months of age and of upper respiratory tract infection at 42 months of age, a girl received a diagnosis of bronchiolitis at 4.5 months of age and of upper respiratory tract infection at 19 months of age, and another girl had an upper respiratory tract infection at both 6 months and 32 months of age.

\section{DISCUSSION}

We used molecular studies and culture to test the hypothesis that human metapneumovirus is a major cause of lower respiratory tract infection in children. We evaluated prospectively acquired respiratory samples from a longitudinal study of children conducted over a period of 
25 years. Human metapneumovirus was present in 20 percent of all cases of lower respiratory tract infection without a prior virologic diagnosis. Extrapolation of these results suggests that 81 human metapneumovirus infections would be expected among the entire group of 408 cases of previously negative lower respiratory tract infection, leading to an overall prevalence in this cohort of 687 children with lower respiratory tract infection of 12 percent. The prevalence of other viruses in this cohort with lower respiratory tract infection was 15 percent for RSV, 10 percent for parainfluenza virus, 5 percent for influenzavirus, and 4 percent for adenovirus. One must be cautious about making direct comparisons of the prevalence of this virus with that of other viruses, which were detected by cell-culture methods. Previous reports suggested that PCR-based diagnostic techniques increase the sensitivity of viral detection. ${ }^{16,17}$ Nonetheless, although the population-based incidence and prevalence cannot be determined from these data, our findings suggest that human metapneumovirus causes lower respiratory tract infection in healthy children at a relatively high frequency. Other reports have noted human metapneumovirus in 4 to 16 percent of specimens obtained from patients with acute respiratory tract infection and submitted to diagnostic virology laboratories. ${ }^{4,18-20}$ Three additional reports on acute respiratory tract infection in adult outpatients noted rates of human metapneumovirus infection of 2 to 7 percent. $7,21,22$ The lower rates found in adults may reflect decreased levels of viral shedding or methodologic differences among the reports.

The demographic features associated with human metapneumovirus infection suggested the classic characteristics of a viral respiratory tract infection of infancy. Male sex was associated with an increased risk of lower respiratory tract disease, as it is for other respiratory viruses. Three quarters of all lower respiratory tract infections caused by human metapneumovirus occurred in the first year of life. Disease due to human metapneumovirus occurred in late winter epidemics that coincided with the latter half of the RSV season. In contrast to a recent report, 23 we did not find evidence of increased severity of disease in children who were coinfected with human metapneumovirus and other viruses, though the number of these children was small.

We also found human metapneumovirus in 15 percent of patients with upper respiratory tract infections. In contrast, human metapneumovirus was detected in only one healthy child, although some of these samples were collected during the summer months, when the virus appears to be less prevalent. Osterhaus and Fouchier ${ }^{22}$ found only one positive specimen among 600 asymptomatic adults and children who were tested. In previous studies at our clinic, adenovirus was isolated from only one of 174 asymptomatic children. ${ }^{9}$ Similarly, viruses were not isolated on day 0 from 68 children in studies of RSV vaccine. ${ }^{24}$

The clinical features of lower respiratory tract infection with human metapneumovirus were similar to those of infections caused by other paramyxoviruses. The statistical association of human metapneumovirus infection with asthma was intriguing in the light of recent conflicting reports regarding a possible association between this infection and asthma. ${ }^{20,25}$ However, the biologic significance of this association is unknown, and asthma is a difficult clinical diagnosis to make in young children.

The limitations of this study include the fact that the use of frozen specimens for RT-PCR and viral culture of human metapneumovirus may have diminished the yield. Although contamination is a concern with the use of highly sensitive techniques such as PCR, we used stringent criteria and sequenced all PCR products, thus reducing the risk of false positive results, but potentially eliminating true positive results that did not meet our criteria. These factors may have led us to underestimate the frequency of respiratory tract infections caused by human metapneumovirus. 
An association between the presence of a virus and symptoms of respiratory tract disease does not necessarily establish causation. Nevertheless, the clinical, demographic, radiographic, and genetic evidence suggests that there is a strong association between human metapneumovirus and lower respiratory tract infection in otherwise healthy children and that human metapneumovirus is a major cause of bronchiolitis and croup in the pediatric population. Lower respiratory tract infections occurred predominantly in the first year of life, during the late winter months. The hospitalization rate was similar to that for RSV infection, and otitis media was a frequent complication. Our data, collected prospectively over a period of 25 years, demonstrate the seasonal occurrence of human metapneumovirus infection and define the spectrum of clinical disease caused by this novel human pathogen.

\section{References}

1. Glezen WP, Loda FA, Clyde WA Jr, et al. Epidemiologic patterns of acute lower respiratory disease of children in a pediatric group practice. J Pediatr 1971;78:397-406. [PubMed: 5101436]

2. Henderson FW, Clyde WA Jr, Collier AM, et al. The etiologic and epidemiologic spectrum of bronchiolitis in pediatric practice. J Pediatr 1979;95:183-90. [PubMed: 448557]

3. Hall CB, Walsh EE, Schnabel KC, et al. Occurrence of groups A and B of respiratory syncytial virus over 15 years: associated epidemiologic and clinical characteristics in hospitalized and ambulatory children. J Infect Dis 1990;162:1283-90. [PubMed: 2230258]

4. van den Hoogen BG, de Jong JC, Groen J, et al. A newly discovered human pneumovirus isolated from young children with respiratory tract disease. Nat Med 2001;7:719-24. [PubMed: 11385510]

5. Peret TC, Boivin G, Li Y, et al. Characterization of human metapneumoviruses isolated from patients in North America. J Infect Dis 2002;185:1660-3. [PubMed: 12023774]

6. Nissen MD, Siebert DJ, Mackay IM, Sloots TP, Withers SJ. Evidence of human metapneumovirus in Australian children. Med J Aust 2002;176:188. [PubMed: 11913922]

7. Stockton J, Stephenson I, Fleming D, Zambon M. Human metapneumovirus as a cause of communityacquired respiratory illness. Emerg Infect Dis 2002;8:897-901. [PubMed: 12194763]

8. Esper F, Boucher D, Weibel C, Martinello RA, Kahn JS. Human metapneumovirus infection in the United States: clinical manifestations associated with a newly emerging respiratory infection in children. Pediatrics 2003;111:1407-10. [PubMed: 12777560]

9. Edwards KM, Thompson J, Paolini J, Wright PF. Adenovirus infections in young children. Pediatrics 1985;76:420-4. [PubMed: 2993992]

10. Reed G, Jewett PH, Thompson J, Tollefson S, Wright PF. Epidemiology and clinical impact of parainfluenza virus infections in otherwise healthy infants and young children $<5$ years old. $J$ Infect Dis 1997;175:807-13. [PubMed: 9086134]

11. Fisher RG, Gruber WC, Edwards KM, et al. Twenty years of outpatient respiratory syncytial virus infection: a framework for vaccine efficacy trials. Pediatrics 1997;99:247-8.abstract

12. Neuzil KM, Zhu Y, Griffin MR, et al. Burden of interpandemic influenza in children younger than 5 years: a 25-year prospective study. J Infect Dis 2002;185:147-52. [PubMed: 11807687]

13. Fan J, Henrickson KJ, Savatski LL. Rapid simultaneous diagnosis of infections with respiratory syncytial viruses $\mathrm{A}$ and $\mathrm{B}$, influenza viruses $\mathrm{A}$ and $\mathrm{B}$, and human parainfluenza virus types 1,2 , and 3 by multiplex quantitative reverse transcription-polymerase chain reaction-enzyme hybridization assay (Hexaplex). Clin Infect Dis 1998;26:1397-402. [PubMed: 9636869]

14. Felsenstein, J. PHYLIP (PHYLogeny Inference Package), version 3.5c. Seattle: Department of Genetics, University of Washington; 1993.

15. Boivin G, Abed Y, Pelletier G, et al. Virological features and clinical manifestations associated with human metapneumovirus: a new paramyxovirus responsible for acute respiratory-tract infections in all age groups. J Infect Dis 2002;186:1330-4. [PubMed: 12402203]

16. Kehl SC, Henrickson KJ, Hua W, Fan J. Evaluation of the Hexaplex assay for detection of respiratory viruses in children. J Clin Microbiol 2001;39:1696-701. [PubMed: 11325976] 
17. Freymuth F, Vabret A, Galateau-Salle F, et al. Detection of respiratory syncytial virus, parainfluenzavirus 3, adenovirus and rhinovirus sequences of respiratory tract of infants by polymerase chain reaction and hybridization. Clin Diagn Virol 1997;8:31-40. [PubMed: 9248656]

18. Mackay IM, Jacob KC, Woolhouse D, et al. Molecular assays for detection of human metapneumovirus. J Clin Microbiol 2003;41:100-5. [PubMed: 12517833]

19. Freymuth F, Vabret A, Legrand L, et al. Presence of the new human metapneumovirus in French children with bronchiolitis. Pediatr Infect Dis J 2003;22:92-4. [PubMed: 12553303]

20. Jartti T, van den Hoogen B, Garafalo RP, Osterhaus AD, Ruuskanen O. Metapneumovirus and acute wheezing in children. Lancet 2002;60:1393-4. [PubMed: 12423987]

21. Falsey AR, Erdman D, Anderson LJ, Walsh EE. Human metapneumovirus infections in young and elderly adults. J Infect Dis 2003;187:785-90. [PubMed: 12599052]

22. Osterhaus A, Fouchier R. Human metapneumovirus in the community. Lancet 2003;361:890-1. [PubMed: 12648965]

23. Greensill J, McNamara PS, Dove W, Flanagan B, Smyth RL, Hart CA. Human metapneumovirus in severe respiratory syncytial virus bronchiolitis. Emerg Infect Dis 2003;9:372-5. [PubMed: 12643835]

24. Wright PF, Karron RA, Belshe RB, et al. Evaluation of a live, cold-passaged, temperature-sensitive, respiratory syncytial virus vaccine candidate in infancy. J Infect Dis 2000;182:1331-42. [PubMed: 11010838]

25. Rawlinson WD, Waliuzzaman Z, Carter IW, Belessis YC, Gilbert KM, Morton JR. Asthma exacerbations in children associated with rhinovirus but not human metapneumovirus infection. $\mathrm{J}$ Infect Dis 2003;187:1314-8. [PubMed: 12696012] 
Human Metapneumovirus

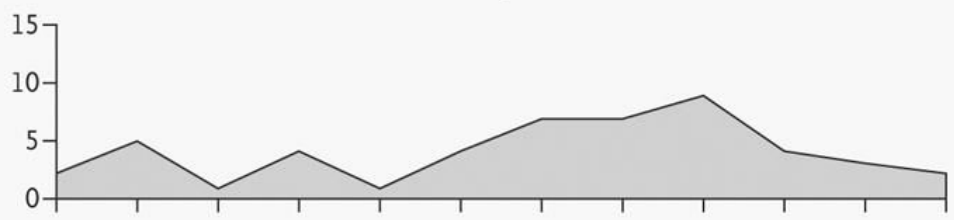

Respiratory Syncytial Virus

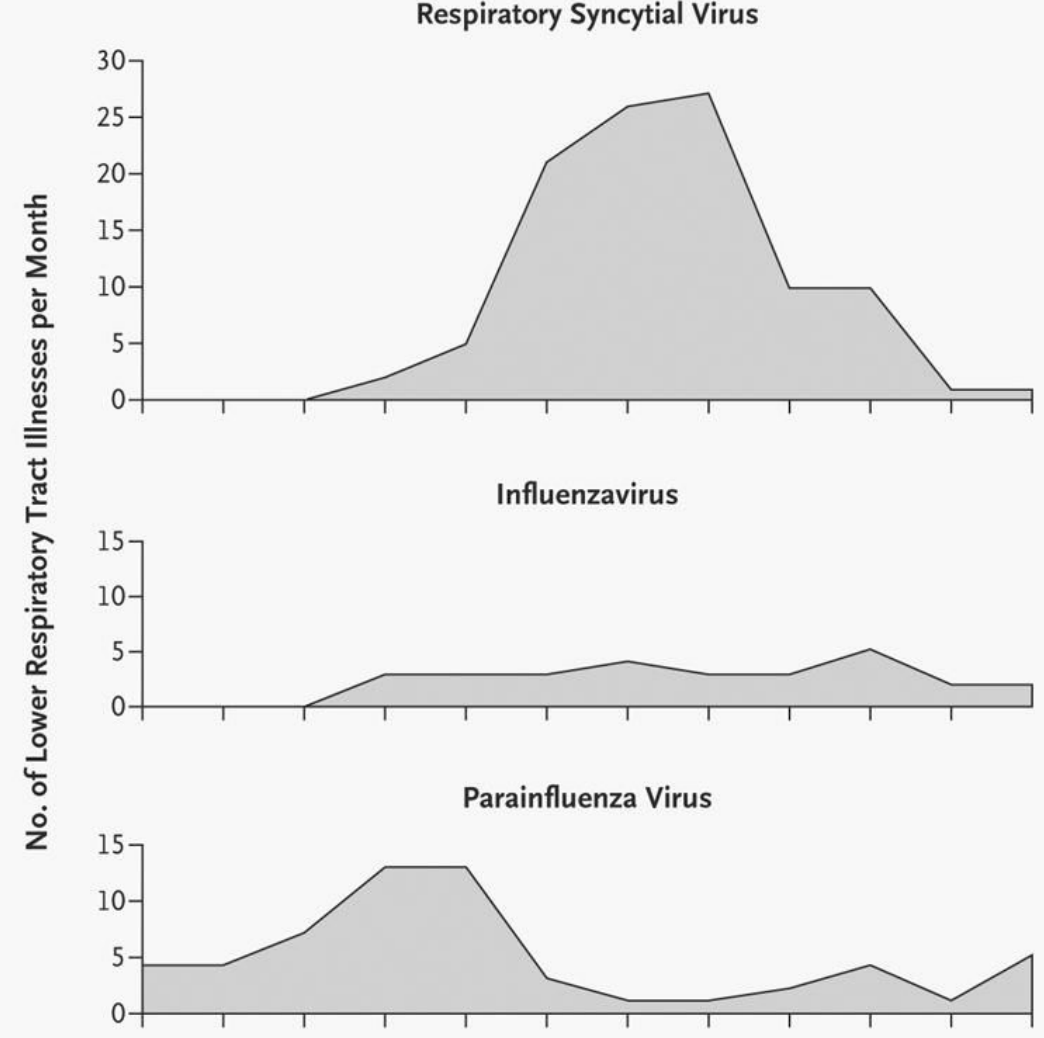

Adenovirus

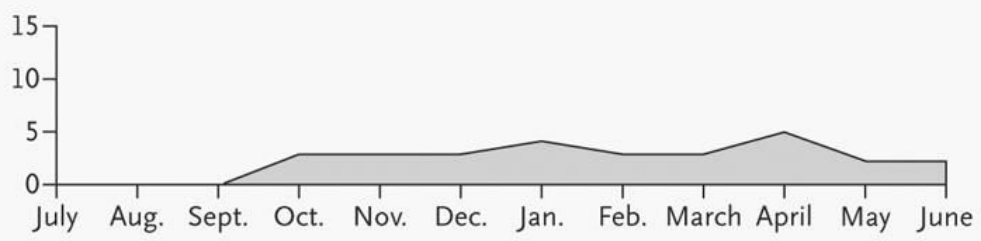

Figure 1. Epidemiologic Pattern of Lower Respiratory Tract Infections with Human Metapneumovirus and Other Viruses

Data are combined from 25 years of surveillance in the Vanderbilt Vaccine Clinic. 


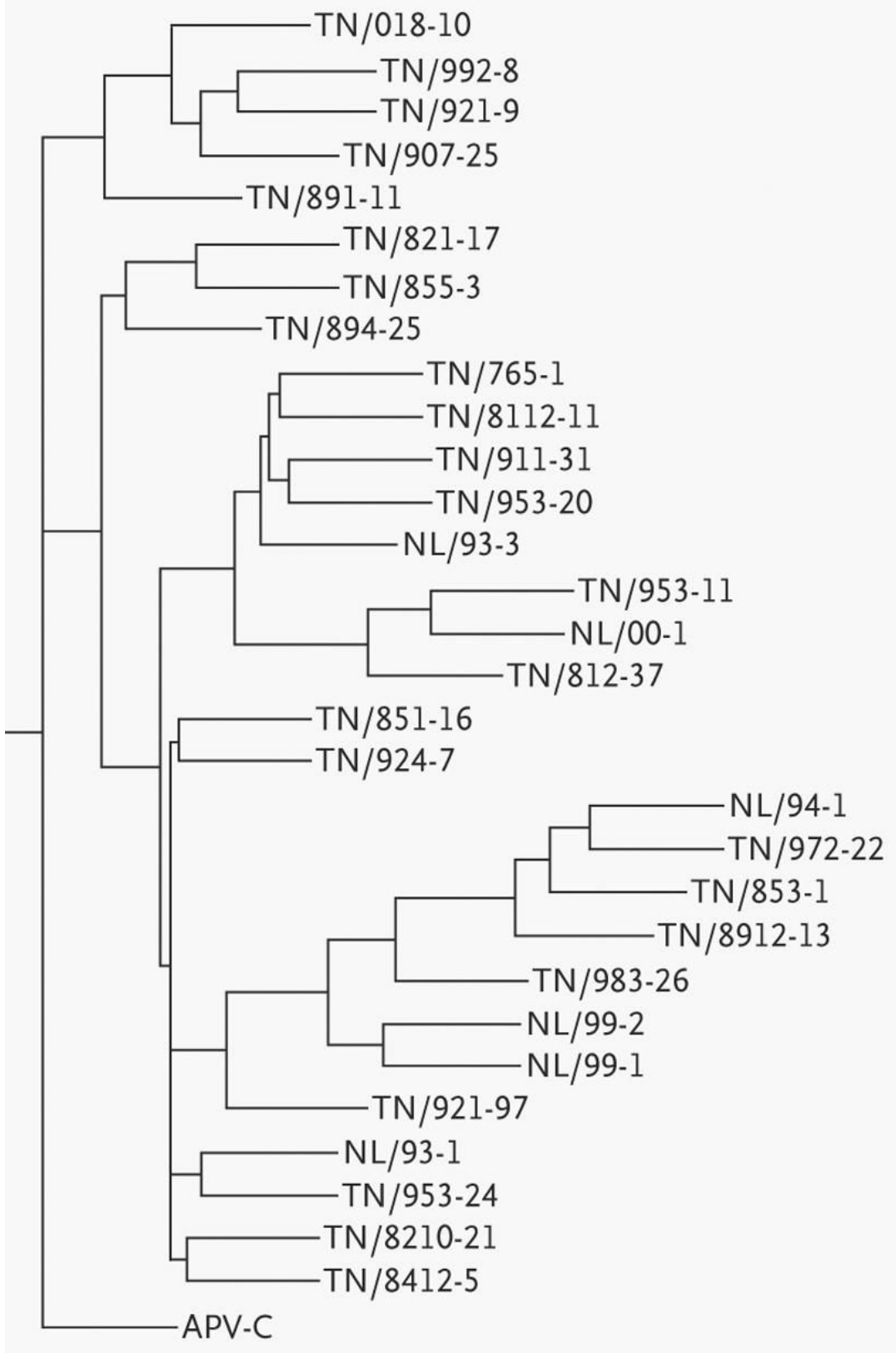

\subsection{Nucleotide substitution per site}

Figure 2. Phylogenetic Tree of Human Metapneumovirus Isolates from Tennessee (TN) and the Netherlands (NL)

The closely related avian pneumovirus (APV-C) was used for comparison. A low frequency of nucleotide substitutions per site (as indicated by the length of the horizontal branch) suggests close genetic relatedness. Two major genotypes are apparent as clusters at the top and bottom of the figure. The first two digits of each TN isolate indicate the year of isolate, the third digit (or third and fourth) the month, and the last digit the number of the sample among all samples collected that month. Sequences are available from GenBank (accession numbers AY216940 to AY216986). 


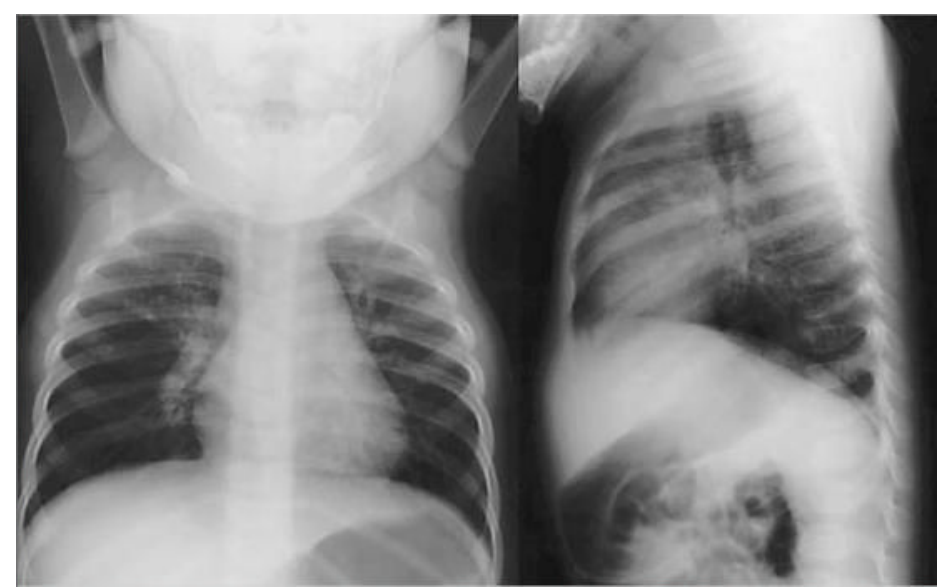

Figure 3. Chest Radiograph Obtained in a Six-Month-Old Infant with Human Metapneumovirus Bronchiolitis

Hyperinflation and diffuse perihilar infiltrates are evident. 
Table 1

Clinical Features of 49 Children with Human Metapneumovirus Infection of the Lower Respiratory Tract.

Feature

Symptoms

Cough

Coryza

Fever

Irritability

Anorexia

Wheezing

Diarrhea

Vomiting

Signs

Rhinitis

Abnormal tympanic membrane

Abnormal tym

Pharyngitis
Rhonchi

Rales
Percent

90

88

52

33

22
17

10

77

52

51

20 


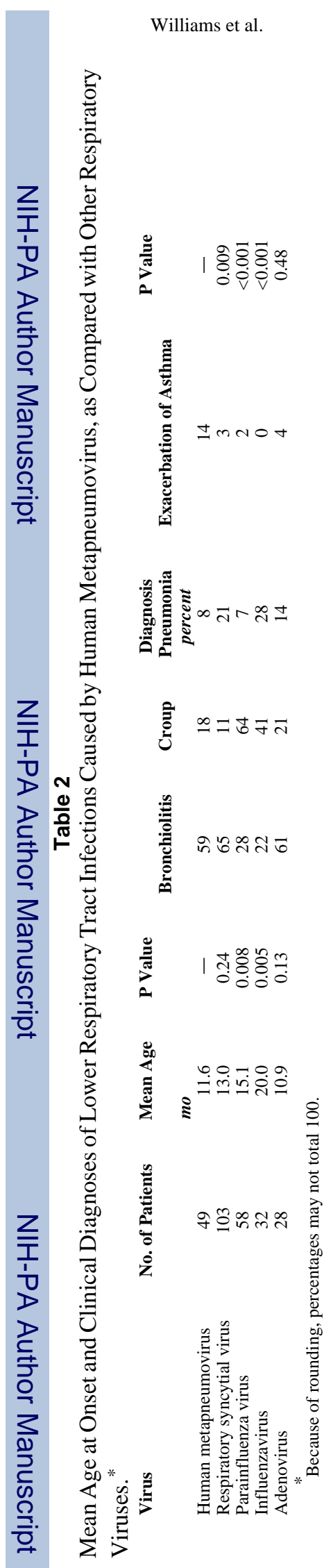

N Engl J Med. Author manuscript; available in PMC 2007 March 26. 